\title{
On the Relationship Between the Stochastic Galerkin Method and the Pseudo-Spectral Collocation Method for Linear Differential Algebraic Equations
}

\author{
Paolo Manfredi • Daniël De Zutter • Dries Vande Ginste
}

Received: date / Accepted: date

\begin{abstract}
Polynomial chaos-based methods have been extensively applied in electrical and other engineering problems for the stochastic simulation of systems with uncertain parameters. Most of the implementations are based on either the intrusive stochastic Galerkin method or on non-intrusive collocation approaches, of which a very common example is the pseudo-spectral method based on Gaussian quadrature rules. This paper shows that, for the important class of linear differential algebraic equations, the latter can be cast as an approximate factorization of the stochastic Galerkin approach, thus generalizing recent discussions in literature in this regard. Consistently with this literature, we show that the factorization turns out to be exact for first-order random inputs, and hence the two methods coincide under this assumption. Further, the presented results also generalize recent work in the field of electrical circuit simulation, in which a similar decomposition was derived ad hoc, via error minimization, for the case of Hermite-chaos. We demonstrate that the factorization stems from the general properties of orthogonal polynomials and the error introduced by the approximation - or in other terms, the error of the stochastic collocation method in comparison with the stochastic Galerkin method — is carefully quantified and assessed. An illustrative example concerning the stochastic analysis of an RLC circuit is used to illustrate the main findings of this paper. In addition, a more complex and real-life example allows emphasizing the generality of the achieved results.
\end{abstract}

Keywords Linear differential algebraic equations · Polynomial chaos · Stochastic collocation method · Stochastic Galerkin method · Matrix factorization · Orthogonal polynomials

\section{Introduction}

Modern applications are facing an increasing impact of technological process variability, e.g., in electrical engineering this is due to the dramatic miniaturization and scaling of device dimensions. The traditional resource to assess the effect of such randomness in the early design phase is to ascribe a suitable distribution to the uncertain and critical design parameters and to perform Monte Carlo analyses [1]. However, Monte Carlo is characterized by a slow convergence rate, which renders the statistical assessment computationally demanding.

For this reason, alternative approaches were recently proposed based on generalized polynomial chaos [2]. According to polynomial chaos, stochastic quantities are expanded into series of suitable orthogonal polynomials, depending on the probability density function $(\mathrm{PDF})$ of the random parameters. The polynomial chaos expansion (PCE) represents an analytical representation from which pertinent statistical information, like stochastic moments or PDFs, is readily derived.

Several methods are available to determine the unknown PCE coefficients [3]. The most accurate approach is the stochastic Galerkin method (SGM) [4], which substitutes the PCE expansions into the original, governing stochastic equations and requires that the residual is orthogonal to the space of the polynomial functions. This procedure is inherently intrusive, i.e., it involves a modification of the problem to be solved and, possibly, also of

This work was supported by the Research Foundation Flanders (FWO) and by the Interuniversity Attraction Poles Program initiated by the Belgian Science Policy Office. Paolo Manfredi is an FWO postdoctoral research fellow.

P. Manfredi

Department of Information Technology, Ghent University/iMinds, Technologiepark-Zwijnaarde 15, 9052 Gent, Belgium

E-mail: paolo.manfredi@ugent.be

D. De Zutter

Department of Information Technology, Ghent University/iMinds, Technologiepark-Zwijnaarde 15, 9052 Gent, Belgium

E-mail: daniel.dezutter@ugent.be

D. Vande Ginste

Department of Information Technology, Ghent University/iMinds, Technologiepark-Zwijnaarde 15, 9052 Gent, Belgium

E-mail: dries.vandeginste@ugent.be 
the solver used to handle the pertinent equations. In fact, it results in a new, deterministic problem of augmented size in the unknown PCE coefficients. The single solution of the new problem is generally much faster than running a large number of Monte Carlo simulations. Recently, this technique has been intensively applied, e.g., in electrical engineering [5-8], civil engineering [9], and fluid dynamics [10].

Alternatively, non-intrusive approaches are represented by the class of collocation methods. The most common one is the pseudo-spectral collocation method (PSCM), which calculates the PCE coefficients according to the classical projection theorem and by means of a numerical integration. Gaussian quadrature rules [11] represent an optimal choice for this integration [3]. The technique has the relevant advantage of requiring only a set of repeated simulations of the original system at the quadrature nodes, and hence, it is straightforward to implement. It has been recently applied, for example, to the analysis of water wave propagation [12]. Galerkin- and collocation-based approaches have been compared from a numerical viewpoint in [13].

The aim of this paper is to determine and assess the relationship between the SGM and the PSCM in the case of linear differential algebraic equations (LDAEs), as often encountered in (electrical) engineering applications. The relationship is established by means of an approximate matrix decomposition, stemming from the general properties of orthogonal polynomials and decoupling the SGM problem into the PSCM one. It was recently proven [14] that these two techniques are equivalent when the random system parameters are represented by firstorder PCEs. This result comes as a special case, as it is shown that the factorization turns out to be exact under the aforementioned assumption. In [15], a similar decomposition was determined ad hoc through visual inspection and error minimization for the specific case of Hermite-chaos, i.e., to model Gaussian variability. In this paper, we generalize and extend this result and we demonstrate that our factorization is valid for arbitrary polynomial basis functions. The error introduced by higher-order random system parameters is also discussed.

The paper is organized as follows. Section 2 introduces the problem and provides the necessary mathematical background. Section 3 outlines the approximate factorization that relates the SGM to the PSCM. Section 4 discusses the error introduced by this approximation. The example of an RLC circuit with uniformly or beta distributed parameters is used to illustrate our generalization in Section 5, together with a more complex and real-life application example dealing with a multiconductor transmission line. Finally, conclusions are drawn in Section 6.

\section{Problem statement and preliminaries}

The following stochastic LDAE is considered:

$$
\mathbf{C}(\xi) \mathbf{x}^{\prime}(t, \xi)+\mathbf{G}(\xi) \mathbf{x}(t, \xi)=\mathbf{u}(t, \xi),
$$

with a suitable initial condition $\mathbf{x}\left(t=t_{0}\right)$. The matrices $\mathbf{C}, \mathbf{G} \in \mathbb{R}^{N \times N}$ and the source term $\mathbf{u} \in \mathbb{R}^{N}$ depend on one non-deterministic and real-valued parameter $\xi \in \mathbb{R}$ with a given PDF $w(\xi)$. The present paper focusses on the derivations for a single random variable, whereas the extension to multiple random variables will be the object of further investigation. It should be noted that the system solution $\mathbf{x}$ is in turn also stochastic as a result of the randomness in the system parameters $\mathbf{C}$ and $\mathbf{G}$.

\subsection{The polynomial chaos expansion (PCE)}

Under the assumption that each component of the solution $\mathbf{x}$ of (1) exhibits finite second-order moments at each time instant $t$, the stochastic system responses are expanded into a truncated PCE as follows:

$$
\mathbf{x}(t, \xi) \approx \sum_{n=0}^{P} \mathbf{x}_{n}(t) p_{n}(\xi)
$$

where $\left\{p_{n}(\xi)\right\}_{n=0}^{P}$ is a basis of polynomials satisfying the orthogonality condition $\left\langle p_{n}, p_{m}\right\rangle=\left\|p_{m}\right\|^{2} \delta_{n m}$, with $\left\|p_{m}\right\|^{2}=\left\langle p_{m}, p_{m}\right\rangle, \delta_{n m}$ the Kronecker's delta, and the inner product being defined as

$$
\langle f(\xi), g(\xi)\rangle=\int_{\mathbb{R}} f(\xi) g(\xi) w(\xi) \mathrm{d} \xi
$$

Depending on the probability distribution $w(\xi)$ of the random parameter $\xi$, a corresponding class of polynomials provides an optimal, exponential convergence rate for the expansion (2). For example, Hermite, Legendre and Jacobi polynomials are the optimal basis functions for Gaussian, uniform and beta distributions, respectively [2]. Letting $P \rightarrow \infty$ yields an exact representation, yet the PCE is truncated in practice. The coefficients $\mathbf{x}_{n}$ are unknown and a suitable technique must be devised for their calculation. Their determination allows statistically quantifying the system response [3].

Usually (see, e.g., [5-8]) a similar PCE is assumed also for the system matrices $\mathbf{G}$ and $\mathbf{C}$, i.e.,

$$
\mathbf{C}(\xi)=\sum_{k=0}^{K} \mathbf{C}_{k} p_{k}(\xi), \quad \mathbf{G}(\xi)=\sum_{k=0}^{K} \mathbf{G}_{k} p_{k}(\xi),
$$


as well as for the source term:

$$
\mathbf{u}(t, \xi)=\sum_{k=0}^{P} \mathbf{u}_{k}(t) p_{k}(\xi)
$$

The coefficients $\mathbf{C}_{k}, \mathbf{G}_{k}$ and $\mathbf{u}_{k}$ are computed, e.g., for $\mathbf{C}_{k}$, as

$$
\mathbf{C}_{k}=\frac{\left\langle\mathbf{C}(\xi), p_{k}(\xi)\right\rangle}{\left\langle p_{k}(\xi), p_{k}(\xi)\right\rangle}=\frac{1}{\left\|p_{k}\right\|^{2}} \int_{\mathbb{R}} \mathbf{C}(\xi) p_{k}(\xi) w(\xi) \mathrm{d} \xi, \quad k=0, \ldots, K
$$

based on the (known) relationship between the system parameters and the random variable $\xi$. For the discussion that follows, it is important to distinguish between the PCE order $K$ of the system matrices in (3) and the PCE order $P$ of the solution in (2). Although it is common practice to take $K=P$, there are relevant cases in which $K$ could actually be lower than $P$. For instance, when the relationship between the system matrices and the random parameter is linear $(K=1)$, of finite order, or when a low order $K$ yields already a satisfactory accuracy in (3).

From now on, we will provide explicit expressions and derivations for the matrix $\mathbf{C}$ only. The results for the matrix $\mathbf{G}$ follow in an identical fashion.

\subsection{Orthogonal polynomials}

Any set of orthogonal polynomials $\left\{p_{n}\right\}_{n=0}^{P}$ satisfies the following three-term recurrence relationship [11]:

$$
p_{n}(\xi)=\left(a_{n} \xi+b_{n}\right) p_{n-1}(\xi)-c_{n} p_{n-2}(\xi), \quad n=1, \ldots, P
$$

with $p_{-1}(\xi) \equiv 0$ and $p_{0}(\xi) \equiv 1$. The degree of the polynomials is thus $\operatorname{deg}\left(p_{n}\right)=n$. The recursion coefficients $a_{n}, b_{n}$ and $c_{n}$ are known for classical orthogonal polynomials that are defined with respect to common and wellknown distributions, e.g., Hermite, Legendre and Jacobi polynomials for Gaussian, uniform and beta distributions, respectively [2]. Suitable coefficients can be numerically determined that yield the required orthogonal polynomials for any arbitrary PDF (see [16] for an application in electrical engineering).

The recurrence relationship (6) is written in matrix form as [11]

$$
\xi \mathbf{p}(\xi)=\mathbf{T} \mathbf{p}(\xi)+\frac{1}{a_{P+1}} p_{P+1}(\xi) \mathbf{e}_{P+1},
$$

where $\mathbf{p}(\xi)=\left[p_{0}(\xi) \ldots p_{P}(\xi)\right]^{t} \in \mathbb{R}^{P+1}$ (the superscript $t$ denoting the transpose operator), $\mathbf{e}_{P+1}=[0 \ldots 01]^{t} \in$ $\mathbb{R}^{P+1}$, whilst $\mathbf{T} \in \mathbb{R}^{(P+1) \times(P+1)}$ is the following tridiagonal matrix:

$$
\mathbf{T}=\left[\begin{array}{ccccc}
-b_{1} / a_{1} & 1 / a_{1} & 0 & \ldots & 0 \\
c_{2} / a_{2} & -b_{2} / a_{2} & 1 / a_{2} & \ldots & \vdots \\
0 & \ddots & \ddots & \ddots & \vdots \\
\vdots & & & & 1 / a_{P} \\
0 & \ldots & \ldots & c_{P+1} / a_{P+1}-b_{P+1} / a_{P+1}
\end{array}\right]
$$

It should be noted that the coefficients $a_{n}$, appearing in (8) at the denominator, are always non-zero. In fact, as inferred from (6), this ensures that all polynomials have different degree, which is a necessary requirement to make them orthogonal.

\subsection{Stochastic Galerkin method}

A common strategy to determine the unknown PCE coefficients is to build a deterministic system of equations by substituting the expansions of the system variables (2), (3) and (4) into (1) and requiring the residual to be orthogonal to the space span by the polynomial basis functions. This leads to

$$
\sum_{k=0}^{K} \sum_{n=0}^{P} \mathbf{C}_{k} \mathbf{x}_{n}^{\prime}\left\langle p_{k}(\xi) p_{n}(\xi), p_{m}(\xi)\right\rangle+\sum_{k=0}^{K} \sum_{n=0}^{P} \mathbf{G}_{k} \mathbf{x}_{n}(t)\left\langle p_{k}(\xi) p_{n}(\xi), p_{m}(\xi)\right\rangle-\left\|p_{m}\right\|^{2} \mathbf{u}_{m}(t)=0, \quad m=0, \ldots, P .
$$

subject to the initial condition $\mathbf{x}_{m}\left(t_{0}\right)=\mathbf{x}\left(t_{0}\right)$ for $m=0$, or $\mathbf{x}_{m}\left(t_{0}\right)=0$ for $m>0$. In (9), all $\xi$-dependence has vanished. Note that the terms $\left\langle p_{k} p_{n}, p_{m}\right\rangle$ and $\left\|p_{m}\right\|^{2}$ are merely real numbers.

Gathering all the $P+1$ equations (9) in matrix form leads to

$$
\widetilde{\mathbf{C}} \widetilde{\mathbf{x}}_{\mathrm{SGM}}^{\prime}(t)+\widetilde{\mathbf{G}} \widetilde{\mathbf{x}}_{\mathrm{SGM}}(t)=\widetilde{\mathbf{u}}(t)
$$


where $\widetilde{\mathbf{x}}_{\mathrm{SGM}}(t)=\left[\mathbf{x}_{0}^{t}(t) \ldots \mathbf{x}_{P}^{t}(t)\right]^{t} \in \mathbb{R}^{(P+1) N}$ collects the unknown PCE coefficients, $\widetilde{\mathbf{u}}(t)=\left[\mathbf{u}_{0}^{t}(t) \ldots \mathbf{u}_{P}^{t}(t)\right]^{t} \in$ $\mathbb{R}^{(P+1) N}$, and $\widetilde{\mathbf{C}}, \widetilde{\mathbf{G}} \in \mathbb{R}^{(P+1) N \times(P+1) N}$ are augmented block matrices, with the $(m, n)$-block of size $N \times N$ given by

$$
\widetilde{\mathbf{C}}_{m n}=\sum_{k=0}^{K} \mathbf{C}_{k} a_{k n m}
$$

where

$$
a_{k n m}=\frac{\left\langle p_{k} p_{n}, p_{m}\right\rangle}{\left\|p_{m}\right\|^{2}}
$$

For some common sets of orthogonal polynomials, closed-form expressions exist for the triple product integral $\left\langle p_{k} p_{n}, p_{m}\right\rangle$ (e.g., see [17] and [18] for Hermite and Legendre polynomials, respectively). According to (11), the matrices $\widetilde{\mathbf{C}}, \widetilde{\mathbf{G}}$ are expressed as

$$
\widetilde{\mathbf{C}}=\sum_{k=0}^{K} \mathbf{A}_{k} \otimes \mathbf{C}_{k}
$$

where the "auxiliary" matrix $\mathbf{A}_{k}$ has entries $A_{k, m n}=a_{k n m}$ and $\otimes$ denotes the Kronecker product.

\subsection{Pseudo-spectral collocation method}

The unknown coefficients $\mathbf{x}_{n}$ can alternatively be computed via the projection theorem:

$$
\mathbf{x}_{n}(t)=\frac{\left\langle\mathbf{x}(t, \xi), p_{n}(\xi)\right\rangle}{\left\langle p_{n}(\xi), p_{n}(\xi)\right\rangle}=\frac{1}{\left\|p_{n}\right\|^{2}} \int_{\mathbb{R}} \mathbf{x}(t, \xi) p_{n}(\xi) w(\xi) \mathrm{d} \xi \approx \frac{1}{\left\|p_{n}\right\|^{2}} \sum_{m=0}^{P} \mathbf{x}\left(t, \xi_{m}\right) p_{n}\left(\xi_{m}\right) w_{m}
$$

Since $\mathbf{x}$ is not available in closed form, the integral in (14) is approximated by a $P$-order Gauss quadrature rule with nodes $\left\{\xi_{m}\right\}_{m=0}^{P}$ and corresponding weights $\left\{w_{m}\right\}_{m=0}^{P}$. The quadrature nodes for this Gauss quadrature are the roots of the polynomial $p_{P+1}(\xi)$. Alternatively, they are also found as the eigenvalues of $\mathbf{T}$ [11], as is easily proven leveraging $(7)$ as follows:

$$
\xi_{m} \mathbf{p}\left(\xi_{m}\right)=\mathbf{T} \mathbf{p}\left(\xi_{m}\right)+\frac{1}{a_{P+1}} \underbrace{p_{P+1}\left(\xi_{m}\right)}_{=0} \mathbf{e}_{P+1}=\mathbf{T} \mathbf{p}\left(\xi_{m}\right), \quad \forall m=0, \ldots, P .
$$

Consequently, $\mathbf{p}\left(\xi_{m}\right)$ is the corresponding eigenvector.

Using (14) requires only a discrete set of evaluations of the system response for the values $\xi_{m}$. This corresponds to asking at the quadrature nodes that:

$$
\mathbf{C}\left(\xi_{m}\right) \mathbf{x}^{\prime}\left(t, \xi_{m}\right)+\mathbf{G}\left(\xi_{m}\right) \mathbf{x}\left(t, \xi_{m}\right)-\mathbf{u}\left(t, \xi_{m}\right)=0, \quad m=0, \ldots, P .
$$

The above $P+1$ equations are now written in matrix form, leading to

$$
\widehat{\mathbf{C}} \widehat{\mathbf{x}}^{\prime}(t)+\widehat{\mathbf{G}} \widehat{\mathbf{x}}(t)=\widehat{\mathbf{u}}(t)
$$

where $\widehat{\mathbf{x}}=\left[\mathbf{x}\left(t, \xi_{0}\right) \ldots \mathbf{x}\left(t, \xi_{P}\right)\right]^{t} \in \mathbb{R}^{(P+1) N}, \widehat{\mathbf{u}}=\left[\mathbf{u}\left(t, \xi_{0}\right) \ldots \mathbf{u}\left(t, \xi_{P}\right)\right]^{t} \in \mathbb{R}^{(P+1) N}$, and $\widehat{\mathbf{C}}, \widehat{\mathbf{G}} \in \mathbb{R}^{(P+1) N \times(P+1) N}$ are block diagonal matrices collecting the system matrices evaluated at the quadrature nodes.

From (14), the vector $\widetilde{\mathbf{x}}_{\mathrm{PSCM}}$, collecting all the unknown PCE coefficients computed by means of the PSCM, is written as $\widetilde{\mathbf{x}}_{\mathrm{PSCM}}=\mathbf{P} \widehat{\mathbf{x}}$, with $\mathbf{P}=\mathbf{Q} \otimes \mathbf{I}_{N}$, where $\mathbf{I}_{N}$ is the $N \times N$ identity matrix and the matrix elements of $\mathbf{Q}$ are given by

$$
Q_{n m}=\frac{p_{n}\left(\xi_{m}\right) w_{m}}{\left\|p_{n}\right\|^{2}}
$$

Substituting the inverse of the above relation into (16) and pre-multiplying both sides by $\mathbf{P}$ yields

$$
\mathbf{P} \widehat{\mathbf{C}} \mathbf{P}^{-1} \widetilde{\mathbf{x}}_{\mathrm{PSCM}}^{\prime}(t)+\mathbf{P} \widehat{\mathbf{G}} \mathbf{P}^{-1} \widetilde{\mathbf{x}}_{\mathrm{PSCM}}(t)=\mathbf{P} \widehat{\mathbf{u}}(t),
$$

where matrix $\mathbf{P}$ is regular due to the orthogonality relation of the polynomials $[14,19]$.

Equation (18) provides an alternative, though equivalent, expression of the PSCM problem, which is now cast in the form (10), i.e., in terms of $\widetilde{\mathbf{x}}_{\mathrm{PSCM}}$ instead of $\widehat{\mathbf{x}}$. The aim of this paper is to assess the relationship between (10) and (18), i.e., between the vectors $\widetilde{\mathbf{u}}$ and $\mathbf{P} \widehat{\mathbf{u}}$, as well as between the matrices $\widetilde{\mathbf{C}}, \widetilde{\mathbf{G}}$ and $\mathbf{P} \widehat{\mathbf{C}} \mathbf{P}^{-1}, \mathbf{P} \widehat{\mathbf{G}} \mathbf{P}^{-1}$. 


\section{The pseudo-spectral method as an approximate factorization of the Galerkin method}

This section shows that the PSCM matrices can be cast as approximate decompositions of the SGM matrices. In particular, the PSCM amounts to approximating the auxiliary matrices $\mathbf{A}_{k}$ by matrix polynomials sharing the same coefficients as the basis functions of the PCE. Furthermore, it is shown that the source terms in the r.h.s. of (10) and (18) also coincide.

Theorem 1 Given the approximation

$$
\xi \mathbf{p}(\xi) \approx \mathbf{T} \mathbf{p}(\xi)
$$

of (7), which neglects the higher-order polynomial $p_{P+1}(\xi)$, the auxiliary matrices in (13) are approximated by a matrix polynomial sharing the same coefficients as the polynomial basis $\left\{p_{n}(\xi)\right\}_{n=0}^{P}$.

Proof Based on the definition (12) of the coefficient $a_{k n m}$, the $k$ th auxiliary matrix is expressed as

$$
\mathbf{A}_{k}=\mathbf{D}\left\langle p_{k}(\xi) \mathbf{p}(\xi), \mathbf{p}^{t}(\xi)\right\rangle,
$$

where $\mathbf{D} \in \mathbb{R}^{(P+1) \times(P+1)}$ is a diagonal matrix with entries $D_{m m}=1 /\left\|p_{m}\right\|^{2}$. Introducing (6) into (20) yields

$$
\mathbf{A}_{k}=a_{k} \mathbf{D}\left\langle\xi p_{k-1}(\xi) \mathbf{p}(\xi), \mathbf{p}^{t}(\xi)\right\rangle+b_{k} \underbrace{\mathbf{D}\left\langle p_{k-1}(\xi) \mathbf{p}(\xi), \mathbf{p}^{t}(\xi)\right\rangle}_{\equiv \mathbf{A}_{k-1}}-c_{k} \underbrace{\mathbf{D}\left\langle p_{k-2}(\xi) \mathbf{p}(\xi), \mathbf{p}^{t}(\xi)\right\rangle}_{\equiv \mathbf{A}_{k-2}} .
$$

So far, the representation is exact. To proceed further, we adopt the approximation (19), which allows us to rewrite $(21)$ as

$$
\mathbf{A}_{k} \approx \check{\mathbf{A}}_{k}=a_{k} \mathbf{D T} \underbrace{\left\langle p_{k-1}(\xi) \mathbf{p}(\xi), \mathbf{p}^{t}(\xi)\right\rangle}_{=\mathbf{D}^{-1} \mathbf{A}_{k-1}}+b_{k} \mathbf{A}_{k-1}-c_{k} \mathbf{A}_{k-2}=\left(a_{k} \mathbf{D T D}^{-1}+b_{k} \mathbf{I}_{P+1}\right) \mathbf{A}_{k-1}-c_{k} \mathbf{A}_{k-2},
$$

The notation $\check{\mathbf{A}}_{k}$ is introduced to distinguish this approximate auxiliary matrix from the exact matrix $\mathbf{A}_{k}$. The impact of this approximation is discussed in Section 4.

If the approximation (19) is now used at every iteration, the exact auxiliary matrices in the r.h.s. of (22) are replaced by the corresponding approximate matrices, leading to

$$
\check{\mathbf{A}}_{k}=\left(a_{k} \mathbf{M}+b_{k} \mathbf{I}_{P+1}\right) \check{\mathbf{A}}_{k-1}-c_{k} \check{\mathbf{A}}_{k-2}, \quad k=1, \ldots, P
$$

where $\mathbf{M}=\mathbf{D T D}^{-1}$, and with $\check{\mathbf{A}}_{-1} \equiv \mathbf{0}$ and $\check{\mathbf{A}}_{0} \equiv \mathbf{A}_{0}=\mathbf{I}_{P+1}$. Equation (23) is formally identical to (6) and defines a recurrence relationship for the approximate matrix $\mathbf{\mathbf { A }}_{k}$ in terms of a matrix polynomial with argument $\mathbf{M}$, sharing the same coefficients $a_{k}, b_{k}$ and $c_{k}$ as the orthogonal polynomial basis.

We may therefore write $\check{\mathbf{A}}_{k}=p_{k}(\mathbf{M})$. Appendix A shows that $\mathbf{M}=\mathbf{T}^{t}$.

Theorem 2 Under the approximation (19) of Theorem 1, the PSCM matrices in the l.h.s. of (18) are a factorization of the SGM matrices in the l.h.s. of (10), i.e.,

$$
\widetilde{\mathbf{C}} \approx \sum_{k=0}^{K} \check{\mathbf{A}}_{k} \otimes \mathbf{C}_{k}=\mathbf{P} \widehat{\mathbf{C}} \mathbf{P}^{-1}
$$

where the approximation of $\widetilde{\mathbf{C}}$ is obtained by replacing in (13) the exact auxiliary matrices $\mathbf{A}_{k}$ with their corresponding approximations $\check{\mathbf{A}}_{k}$.

Proof First, consider the eigendecomposition $\mathbf{M}=\mathbf{V} \boldsymbol{\Lambda} \mathbf{V}^{-1}$. Since $\mathbf{M}=\mathbf{T}^{t}$, these two matrices share the same eigenvalues $\xi_{m}$. Furthermore, as $\mathbf{M}=\mathbf{D} \mathbf{T} \mathbf{D}^{-1}$, given $\mathbf{V}_{m}$ an eigenvector of $\mathbf{M}$, then $\mathbf{D}^{-1} \mathbf{V}_{m}$ is the corresponding eigenvector of $\mathbf{T}$. Of course, the diagonal entries of $\boldsymbol{\Lambda}$ are $\Lambda_{m m}=\xi_{m}$. By further considering that $\mathbf{M}^{k}=\mathbf{V} \boldsymbol{\Lambda}^{k} \mathbf{V}^{-1}$, it follows that

$$
\check{\mathbf{A}}_{k}=p_{k}(\mathbf{M})=\mathbf{V} p_{k}(\boldsymbol{\Lambda}) \mathbf{V}^{-1}
$$

with the entries $\left[p_{k}(\boldsymbol{\Lambda})\right]_{m m}=p_{k}\left(\xi_{m}\right), m=0, \ldots, P$. Substituting (25) into the 1.h.s. of (24) yields

$$
\sum_{k=0}^{K} \check{\mathbf{A}}_{k} \otimes \mathbf{C}_{k}=\mathbf{B}\left(\sum_{k=0}^{K} p_{k}(\boldsymbol{\Lambda}) \otimes \mathbf{C}_{k}\right) \mathbf{B}^{-1}
$$

with $\mathbf{B}=\mathbf{V} \otimes \mathbf{I}_{N}$ and $\mathbf{B}^{-1}=\mathbf{V}^{-1} \otimes \mathbf{I}_{N}$

Second, given the form (3) of the matrix $\mathbf{C}$, it follows immediately that the PSCM matrices described in (16) are given by

$$
\sum_{k=0}^{K} p_{k}(\boldsymbol{\Lambda}) \otimes \mathbf{C}_{k}=\widehat{\mathbf{C}}
$$


and thus

$$
\sum_{k=0}^{K} \check{\mathbf{A}}_{k} \otimes \mathbf{C}_{k}=\mathbf{B} \widehat{\mathbf{C}} \mathbf{B}^{-1}
$$

(and similarly for $\widetilde{\mathbf{G}}$ ).

Finally, to complete the proof, comparing (26) with $\mathbf{P} \widehat{\mathbf{C}} \mathbf{P}^{-1}$ in (18) and (24), the equivalence of matrices $\mathbf{P}$ and $\mathbf{B}$ is still to be assessed. This is readily carried out by noting that $\mathbf{Q}$, like $\mathbf{V}$, is also a matrix of eigenvectors of $\mathbf{M}$. In fact, from (17), the $m$ th column of $\mathbf{Q}$ is expressed as $\mathbf{Q}_{m}=\mathbf{D} \mathbf{p}\left(\xi_{m}\right) w_{m}$. Since, from $(15)$, $\mathbf{p}\left(\xi_{m}\right)$ is an eigenvector of $\mathbf{T}$, then $\mathbf{D} \mathbf{p}\left(\xi_{m}\right)$ is an eigenvector of $\mathbf{M}$. This also holds for its rescaled version $\mathbf{Q}_{m}$. Hence, the factorization of the approximate SGM matrices (24) coincides with the PSCM matrices in (18).

There remains to assess the relationship between $\widetilde{\mathbf{u}}$ and $\mathbf{P} \widehat{\mathbf{u}}$.

Theorem 3 Given the representation (4) of the vector of independent stimuli, then $\widetilde{\mathbf{u}}=\mathbf{P} \widehat{\mathbf{u}}$.

Proof According to the definition of the matrix $\mathbf{P}$, the $n$th block of $\mathbf{P} \widehat{\mathbf{u}}$ is given by

$$
[\mathbf{P} \widehat{\mathbf{u}}]_{n}=\sum_{m=0}^{P} \frac{p_{n}\left(\xi_{m}\right) w_{m}}{\left\|p_{n}\right\|^{2}} \mathbf{u}\left(t, \xi_{m}\right)
$$

By definition of Gauss quadrature,

$$
\sum_{m=0}^{P} \mathbf{u}\left(t, \xi_{m}\right) p_{n}\left(\xi_{m}\right) w_{m}=\int_{\mathbb{R}} \mathbf{u}(t, \xi) p_{n}(\xi) w(\xi) \mathrm{d} \xi=\sum_{k=0}^{P} \mathbf{u}_{k}(t) \underbrace{\int_{\mathbb{R}} p_{k}(\xi) p_{n}(\xi) w(\xi) \mathrm{d} \xi}_{\left\|p_{n}\right\|^{2} \delta_{k n}}=\left\|p_{n}\right\|^{2} \mathbf{u}_{n}(t),
$$

The quadrature is exact since the integrand is a polynomial of degree $\leq 2 P$. Hence, $[\mathbf{P} \widehat{\mathbf{u}}]_{n}=\mathbf{u}_{n}(t)$, i.e., the $n$th block of $\mathbf{P} \widehat{\mathbf{u}}$ coincides with the $n$th PCE coefficient of $\mathbf{u}$. Since $\widetilde{\mathbf{u}}=\left[\mathbf{u}_{0}^{t} \ldots \mathbf{u}_{P}^{t}\right]^{t}$, applying the consideration $\forall n=0, \ldots, P$ yields the complete proof.

In summary, the PSCM coincides with an approximation of the SGM, where the pertinent augmented matrices are constructed by replacing the auxiliary matrices $\mathbf{A}_{k}$ in (13) with the approximate matrices $\check{\mathbf{A}}_{k}$, given by (25) as matrix polynomials that share the same coefficients as used in the polynomial chaos basis.

\section{Error assessment}

\subsection{Error on the auxiliary matrices}

The error between the exact auxiliary matrix $\mathbf{A}_{k}$ and its approximation $\check{\mathbf{A}}_{k}$ is introduced by neglecting the term $\frac{1}{a_{P+1}} p_{P+1}(\xi) \mathbf{e}_{P+1}$ in the substitution of (7) into (21). By taking the above term into account, we may write $\mathbf{A}_{k}=\check{\mathbf{A}}_{k}+\boldsymbol{\epsilon}_{k}^{\prime}$, with

$$
\boldsymbol{\epsilon}_{k}^{\prime}=\frac{a_{k}}{a_{P+1}} \mathbf{D} \mathbf{e}_{P+1}\left\langle p_{k-1}(\xi) p_{P+1}(\xi), \mathbf{p}^{t}(\xi)\right\rangle
$$

It is important to note that the error matrix (27) solely represents the error introduced in (22), i.e., at the $k$ th iteration, and only its last row is non-zero. If $\check{\mathbf{A}}_{k}$ is computed as a matrix polynomial, i.e., by using in fact (23) instead of (22), the errors introduced at the previous iterations must be suitably propagated. By denoting as $\boldsymbol{\epsilon}_{k}$ the total error on matrix $\check{\mathbf{A}}_{k}$, the exact matrix is expressed as

$$
\mathbf{A}_{k}=\left(a_{k} \mathbf{M}+b_{k} \mathbf{I}_{P+1}\right)\left(\check{\mathbf{A}}_{k-1}+\boldsymbol{\epsilon}_{k-1}\right)-c_{k}\left(\check{\mathbf{A}}_{k-2}+\boldsymbol{\epsilon}_{k-2}\right)+\boldsymbol{\epsilon}_{k}^{\prime}=\check{\mathbf{A}}_{k}+\boldsymbol{\epsilon}_{k}^{\prime}+\left(a_{k} \mathbf{M}+b_{k} \mathbf{I}_{P+1}\right) \boldsymbol{\epsilon}_{k-1}-c_{k} \boldsymbol{\epsilon}_{k-2},
$$

with a total error on the $k$ th auxiliary matrix given by

$$
\boldsymbol{\epsilon}_{k}=\frac{a_{k}}{a_{P+1}} \mathbf{D} \mathbf{e}_{P+1}\left\langle p_{k-1}(\xi) p_{P+1}(\xi), \mathbf{p}^{t}(\xi)\right\rangle+\left(a_{k} \mathbf{M}+b_{k} \mathbf{I}\right) \boldsymbol{\epsilon}_{k-1}-c_{k} \boldsymbol{\epsilon}_{k-2} .
$$

The total error is thus the sum of the error $\epsilon_{k}^{\prime}$ introduced at the current iteration and the propagation of the total errors $\epsilon_{k-1}$ and $\epsilon_{k-2}$ at the two previous iterations.

Lemma 1 The total error matrix $\boldsymbol{\epsilon}_{1}$ is always zero.

Proof The inner product in the error expression (27) is given by

$$
\left\langle p_{0}(\xi) p_{P+1}(\xi), \mathbf{p}^{t}(\xi)\right\rangle=\left\langle p_{P+1}(\xi), \mathbf{p}^{t}(\xi)\right\rangle,
$$

as $p_{0}(\xi)=1$. The above inner product is always zero due to the orthogonality of the polynomials. Hence, $\boldsymbol{\epsilon}_{1}^{\prime}=\mathbf{0}$. Since by definition $\check{\mathbf{A}}_{0}=\mathbf{A}_{0}=\mathbf{I}_{P+1}$, and therefore $\boldsymbol{\epsilon}_{0}=\mathbf{0}$, we have that $\boldsymbol{\epsilon}_{1}=\boldsymbol{\epsilon}_{1}^{\prime}=\mathbf{0}$. 


\subsection{Error on the SGM matrices}

The resulting error in the factorization (24) of the SGM matrices is now readily found by substituting $\mathbf{A}_{k}=\check{\mathbf{A}}_{k}+\boldsymbol{\epsilon}_{k}$ into (13):

$$
\widetilde{\mathbf{C}}=\sum_{k=0}^{K} \mathbf{A}_{k} \otimes \mathbf{C}_{k}=\sum_{k=0}^{K}\left(\check{\mathbf{A}}_{k}+\boldsymbol{\epsilon}_{k}\right) \otimes \mathbf{C}_{k}=\sum_{k=0}^{K} \check{\mathbf{A}}_{k} \otimes \mathbf{C}_{k}+\widetilde{\mathbf{E}}_{C}
$$

with

$$
\widetilde{\mathbf{E}}_{C}=\sum_{k=0}^{K} \boldsymbol{\epsilon}_{k} \otimes \mathbf{C}_{k}
$$

Owing to the properties of matrix norm and Kronecker product, an upper bound of the error norm is given by

$$
\left\|\widetilde{\mathbf{E}}_{C}\right\| \leq \sum_{k=0}^{K}\left\|\boldsymbol{\epsilon}_{k}\right\| \cdot\left\|\mathbf{C}_{k}\right\|
$$

Theorem 4 If the random system matrices $\mathbf{C}(\xi)$ and $\mathbf{G}(\xi)$ are represented by first-order PCEs, i.e., $K=1$ in (3), then the SGM problem coincides with the PSCM problem.

The proof readily follows by noting that the approximation errors $\widetilde{\mathbf{E}}_{C}$ and $\widetilde{\mathbf{E}}_{G}$ on the factorized SGM matrices are zero since, according to Lemma $1, \boldsymbol{\epsilon}_{0}=\boldsymbol{\epsilon}_{1}=\mathbf{0}$. It is worth noting that Theorem 4 is equivalent to Theorem 2 in [14], which relies on the fact that under this assumption the Gauss quadrature utilized by the PSCM reproduces exactly the projection integrals in (12).

More in general, equation (28) quantifies the error that exists for $K>1$ between the matrices defining the SGM and the PSCM problems or, alternatively, the error arising with the PSCM in the computation of the projections in (12). It should be noted that, since the PCE coefficients $\mathbf{C}_{k}$ exponentially decay to zero as the order $K$ is increased [2], the above error is expected to converge to a constant value. The numerical results provided in the following section confirm that this is indeed the case.

The results in this section can be readily extended to the case of multiple random variables, provided that a full tensor-product basis of polynomials is chosen (see $[14,15]$ ). Analogous derivations for more common (e.g., total degree) truncation schemes are currently under investigation.

\section{Results and discussion}

5.1 RLC circuit

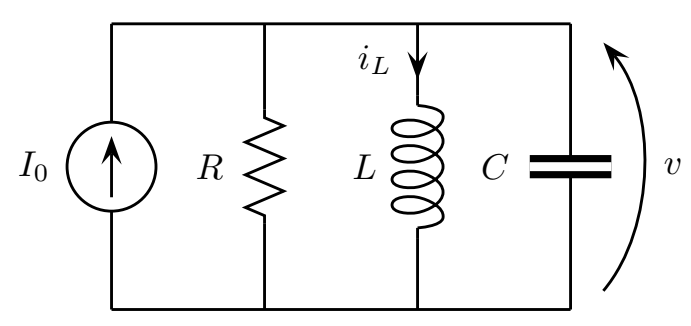

Fig. 1 Schematic of the RLC circuit.

Consider the RLC circuit depicted in Figure 1. The pertinent LDAE is here the modified nodal analysis (MNA) equation [20], given by

$$
\left(\begin{array}{cc}
C & 0 \\
0 & -L
\end{array}\right)\left(\begin{array}{c}
v^{\prime} \\
i_{L}^{\prime}
\end{array}\right)+\left(\begin{array}{cc}
G & 1 \\
1 & 0
\end{array}\right)\left(\begin{array}{c}
v \\
i_{L}
\end{array}\right)=\left(\begin{array}{c}
I_{0} \\
0
\end{array}\right)
$$

with $G=1 / R$. We assume $I_{0}=5 \mathrm{~A}$, the initial condition $\left[v\left(t_{0}\right), i_{L}\left(t_{0}\right)\right]^{t}=[0,0]^{t}$, with $t_{0}=0$, and the following dependencies of the element values on the random variable $\xi$ :

$$
R(\xi)=\bar{R}\left(1+\Delta_{R} \xi\right) \quad, \quad L(\xi)=\frac{\bar{L}}{1+\Delta_{L} \xi} \quad, \quad C(\xi)=\frac{\bar{C}}{1+\Delta_{C} \xi}
$$

with $\bar{R}=10 \Omega, \Delta_{R}=80 \%, \bar{L}=100 \mathrm{nH}, \Delta_{L}=60 \%, \bar{C}=1 \mathrm{nF}, \Delta_{C}=70 \%$. The PCE coefficients of the MNA system matrices in (3) are given by

$$
\mathbf{C}_{k}=\left(\begin{array}{cc}
C_{k} & 0 \\
0 & -L_{k}
\end{array}\right) \forall k \quad, \quad \mathbf{G}_{k}=\left\{\begin{array}{l}
\left(\begin{array}{cc}
G_{0} & 1 \\
1 & 0
\end{array}\right) k=0 \\
\left(\begin{array}{cc}
G_{k} & 0 \\
0 & 0
\end{array}\right) k>0
\end{array}\right.
$$


with

$$
G_{k}=\frac{1}{\left\|p_{k}\right\|^{2}} \int_{\mathbb{R}} \frac{1}{R(\xi)} p_{k}(\xi) w(\xi) \mathrm{d} \xi \quad, \quad L_{k}=\frac{1}{\left\|p_{k}\right\|^{2}} \int_{\mathbb{R}} L(\xi) p_{k}(\xi) w(\xi) \mathrm{d} \xi \quad, \quad C_{k}=\frac{1}{\left\|p_{k}\right\|^{2}} \int_{\mathbb{R}} C(\xi) p_{k}(\xi) w(\xi) \mathrm{d} \xi
$$

\subsubsection{Uniformly distributed parameters}
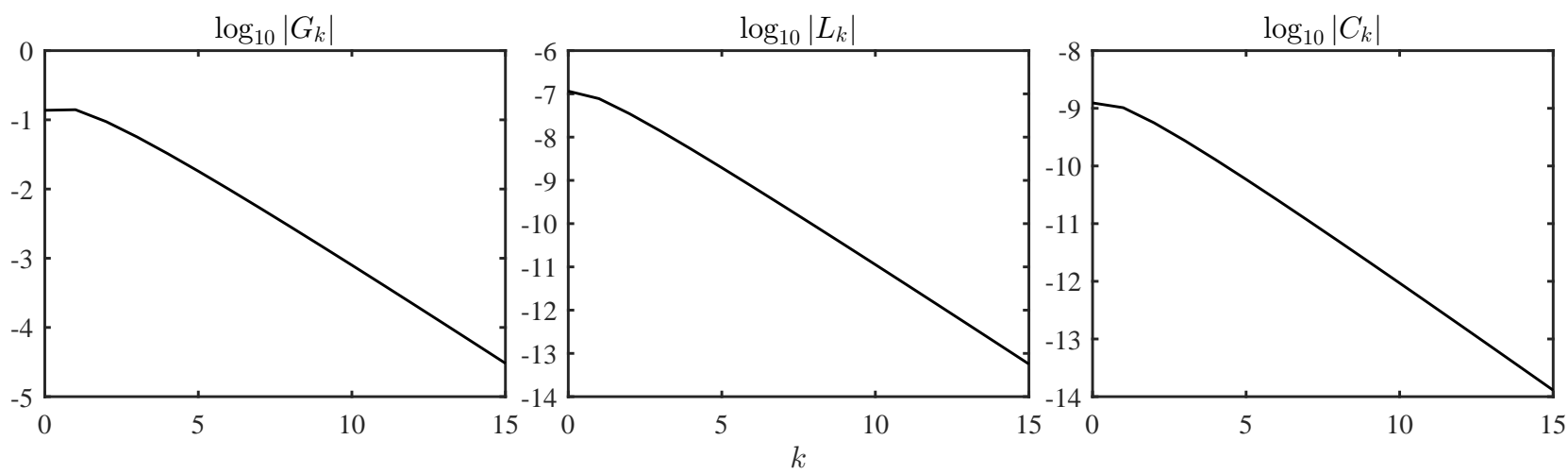

Fig. 2 PCE coefficients of the circuit parameters with uniform variability.

Let the random variable $\xi$ assume a uniform distribution in the interval $[-1,1]$. Therefore, $w(\xi)=\frac{1}{2}$ for $|\xi| \leq 1$ and the orthogonal functions are the Legendre polynomials. The coefficients of their recurrence relationship (6) are

$$
\begin{aligned}
& a_{n}=(2 n-1) / n \\
& c_{n}=(n-1) / n
\end{aligned}
$$

and the first five polynomials are

$$
\begin{aligned}
& p_{0}(\xi)=1 \\
& p_{1}(\xi)=\xi \\
& p_{2}(\xi)=\frac{3}{2} \xi^{2}-\frac{1}{2} \\
& p_{3}(\xi)=\frac{5}{2} \xi^{3}-\frac{3}{2} \xi \\
& p_{4}(\xi)=\frac{35}{8} \xi^{4}-\frac{30}{8} \xi^{2}+\frac{3}{8}
\end{aligned}
$$

Figure 2 shows the PCE coefficients of the element values calculated up to order $K=15$ by solving (5) via an adaptive Gauss-Kronrod quadrature. As expected, these coefficients exhibit an exponential decay rate.

The expressions of the approximate auxiliary matrices $\check{\mathbf{A}}_{k}$ that define the PSCM problem for a fourth-order analysis $(P=4)$ are

$$
\begin{aligned}
\check{\mathbf{A}}_{0} & \equiv \mathbf{A}_{0}=\mathbf{I}_{5} \\
\check{\mathbf{A}}_{1} & \equiv \mathbf{A}_{1}=\mathbf{M} \\
\check{\mathbf{A}}_{2} & =\frac{3}{2} \cdot \mathbf{M}^{2}-\frac{1}{2} \cdot \mathbf{I}_{5} \\
\check{\mathbf{A}}_{3} & =\frac{5}{2} \cdot \mathbf{M}^{3}-\frac{3}{2} \cdot \mathbf{M} \\
\check{\mathbf{A}}_{4} & =\frac{35}{8} \cdot \mathbf{M}^{4}-\frac{30}{8} \cdot \mathbf{M}^{2}+\frac{3}{8} \cdot \mathbf{I}_{5}
\end{aligned}
$$

As a result of Theorem 1, these are given in terms of a polynomial with the same coefficients as the Legendre basis functions, with argument

$$
\mathbf{M}=\mathbf{T}^{t}=\left[\begin{array}{ccccc}
0 & \frac{1}{3} & & & \\
1 & 0 & \frac{2}{5} & & \\
& \frac{2}{3} & 0 & \frac{3}{7} & \\
& & \frac{3}{5} & 0 & \frac{4}{9} \\
& & & \frac{4}{7} & 0
\end{array}\right]
$$


where $\mathbf{T}$ is the tridiagonal matrix in (8).

The corresponding PCE coefficients of the circuit responses are computed for different PCE orders of the MNA matrices, from $K=1$ to $K=4$. Figure 3 shows the time evolution of the PCE coefficients of the voltage $v$ across the capacitor, computed with both the SGM (solid lines) and the PSCM (markers). The plots confirm that the results of the two methods only coincide perfectly when $K=1$.

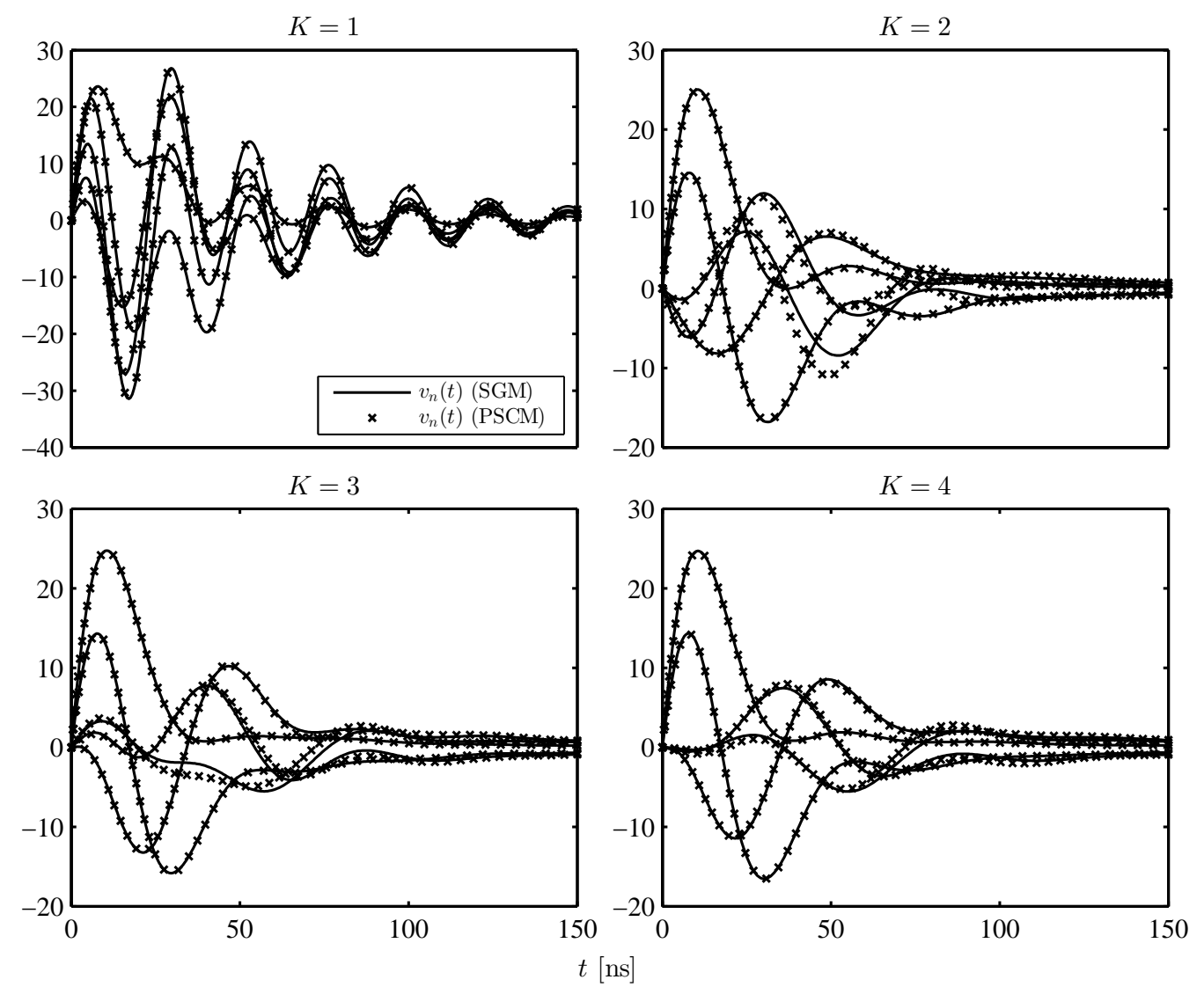

Fig. 3 Transient PCE coefficients $v_{n}(n=0,1,2,3,4)$ of the voltage $v$ across the capacitor in the circuit of Figure 1, computed with the SGM (solid lines) and PSCM (markers) for uniform variability and different expansion orders of the system matrices.

\subsubsection{Beta distributed parameters}
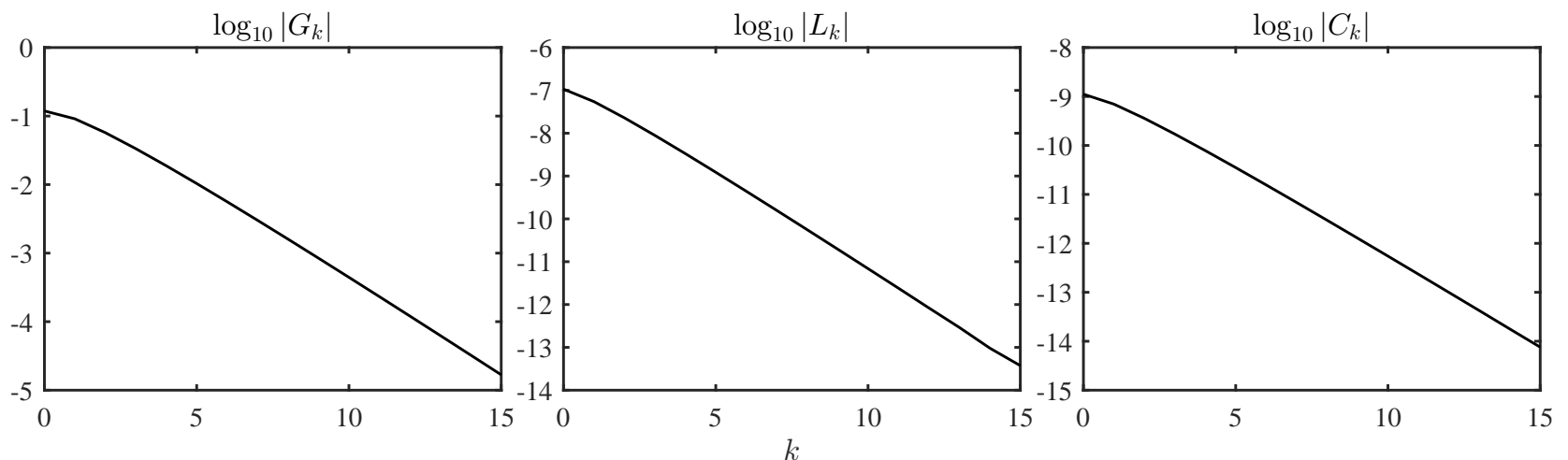

Fig. 4 PCE coefficients of the circuit parameters with beta variability.

Similar results are provided for the case in which $\xi$ has a beta distribution in the interval $[-1,1]$, i.e.,

$$
w(\xi)=\frac{(\xi+1)^{\beta}(1-\xi)^{\alpha}}{2^{\alpha+\beta+1} B(\alpha+1, \beta+1)}
$$


with $B(x, y)$ the beta function and $\alpha, \beta>-1$ being two parameters that affect the shape of the distribution. The functions to be used in the PCEs are the Jacobi polynomials, whose recurrence relationship coefficients are given by

$$
\begin{aligned}
a_{1} & =\frac{1}{2} \cdot \frac{\Gamma(\alpha+\beta+3)}{\Gamma(\alpha+\beta+2)} \\
b_{1} & =\frac{\Gamma(\alpha+2)}{\Gamma(\alpha+1)}-\frac{1}{2} \cdot \frac{\Gamma(\alpha+\beta+3)}{\Gamma(\alpha+\beta+2)}
\end{aligned}
$$

for $n=1$, and by

$$
\begin{aligned}
a_{n} & =\frac{(2 n+\alpha+\beta-1)(2 n+\alpha+\beta)}{2 n(n+\alpha+\beta)} \\
b_{n} & =\frac{\left(\alpha^{2}-\beta^{2}\right)(2 n+\alpha+\beta-1)}{2 n(n+\alpha+\beta)(2 n+\alpha+\beta-2)} \\
c_{n} & =\frac{2(n+\alpha-1)(n+\beta-1)(2 n+\alpha+\beta)}{2 n(n+\alpha+\beta)(2 n+\alpha+\beta-2)}
\end{aligned}
$$

for $n>1$. Considering $\alpha=0.2$ and $\beta=0.4$, the corresponding first five Jacobi polynomials are

$$
\begin{aligned}
& p_{0}(\xi)=1 \\
& p_{1}(\xi)=1.3 \xi-0.1 \\
& p_{2}(\xi)=2.07 \xi^{2}-0.18 \xi-0.57 \\
& p_{3}(\xi)=3.542 \xi^{3}-0.322 \xi^{2}-1.886 \xi+0.074 \\
& p_{4}(\xi)=6.2909 \xi^{4}-0.5852 \xi^{3}-4.9434 \xi^{2}+0.2772 \xi+0.4389
\end{aligned}
$$

Figure 4 shows the pertinent PCE coefficients of the circuit elements computed up to order $K=15$. The approximate auxiliary matrices that define the PSCM for a fourth-order analysis are

$$
\begin{aligned}
& \check{\mathbf{A}}_{0} \equiv \mathbf{A}_{0}=\mathbf{I}_{5} \\
& \check{\mathbf{A}}_{1} \equiv \mathbf{A}_{1}=1.3 \cdot \mathbf{M}-0.1 \cdot \mathbf{I}_{5} \\
& \check{\mathbf{A}}_{2}=2.07 \cdot \mathbf{M}^{2}-0.18 \cdot \mathbf{M}-0.57 \cdot \mathbf{I}_{5} \\
& \check{\mathbf{A}}_{3}=3.542 \cdot \mathbf{M}^{3}-0.322 \cdot \mathbf{M}^{2}-1.886 \cdot \mathbf{M}+0.074 \cdot \mathbf{I}_{5} \\
& \check{\mathbf{A}}_{4}=6.2909 \cdot \mathbf{M}^{4}-0.5852 \cdot \mathbf{M}^{3}-4.9434 \cdot \mathbf{M}^{2}+0.2772 \cdot \mathbf{M}+0.4389 \cdot \mathbf{I}_{5}
\end{aligned}
$$

Also for this case, they are expressed as polynomials sharing the same coefficients as the corresponding Jacobi basis functions, with argument

$$
\mathbf{M}=\mathbf{T}^{t}=\left[\begin{array}{lllll}
0.0769 & 0.3590 & & & \\
0.7692 & 0.0100 & 0.4099 & & \\
& 0.6280 & 0.0040 & 0.4338 & \\
& & 0.5844 & 0.0021 & 0.4477 \\
& & & 0.5630 & 0.0013
\end{array}\right]
$$

Figure 5 shows the PCE coefficients of the current $i_{L}$ flowing through the inductor, computed with the SGM and PSCM methods for different PCE orders of the MNA matrices. Once again, and as expected, the solutions of the two methods coincide only when $K=1$.

\subsubsection{Error and discussion}

In order to assess the impact of the PCE order of the system matrices, the order of the analysis is now fixed to $P=15$ and the relative error between the exact and approximate auxiliary matrices, as well as between the SGM and PSCM matrices is calculated for increasing $K$ and collected in Table 1 . The results in the table show that an error always exists between the SGM and the PSCM matrices when $K \neq 1$. This causes the difference observed in Figures 3 and 5 between the PCE coefficients obtained by means of two techniques when $K>1$. The error eventually converges to a specific value due to the exponential decay rate of the coefficients in (28). More specifically, the results of the SGM and the PSCM rigorously coincide only if $K=1$, even for large values of $P$. Nevertheless, the exponential decay of the coefficients also causes the relative error between the SGM and PSCM matrices to be considerably lower than the error on the auxiliary matrices, and makes the difference between the SGM and the PSCM negligible in practical applications with moderate variability of the system parameters.

This simple example allowed illustrating the main findings of this paper, namely the polynomial expression of the approximate auxiliary matrices that relate the SGM and the PSCM, the equivalence between these two methods for first-order system matrices, and the overall behavior of the associated error. 

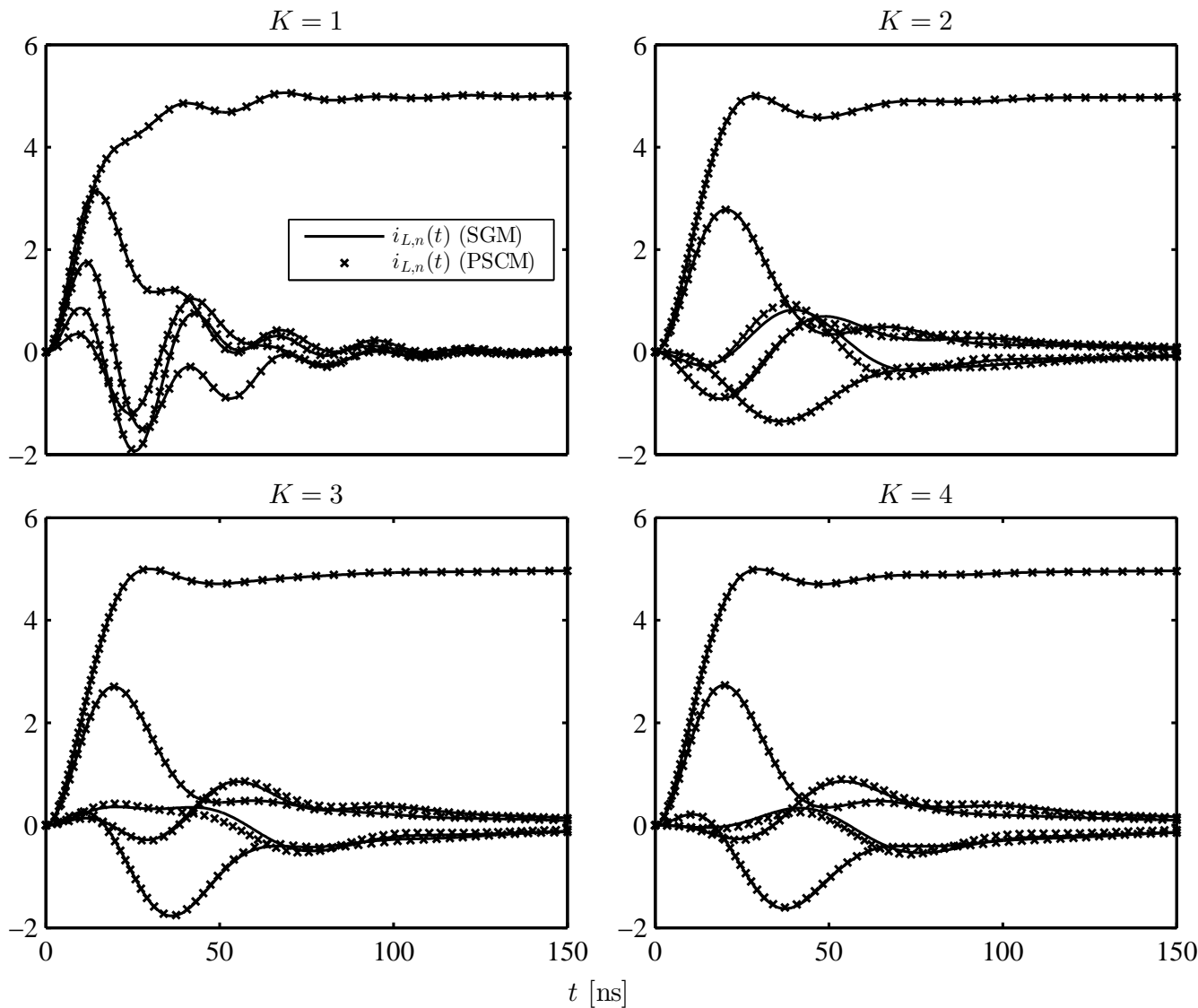

Fig. 5 Transient PCE coefficients $i_{L, n}(n=0,1,2,3,4)$ of the current $i_{L}$ flowing through the inductor in the circuit of Figure 1 , computed with the SGM (solid lines) and PSCM (markers) for beta variability and different expansion orders of the system matrices.

Table 1 Error between the exact and approximate auxiliary matrices and corresponding error on the SGM and PSCM matrices for a fifteenth-order analysis $(P=15)$ and different expansion orders of the system matrices.

\begin{tabular}{c|ccc|ccc|} 
& \multicolumn{3}{|c|}{ uniform variability } & \multicolumn{3}{c|}{ beta variability } \\
\hline$K$ & $\left\|\boldsymbol{\epsilon}_{K}\right\| /\left\|\mathbf{A}_{K}\right\|$ & $\left\|\tilde{\mathbf{E}}_{C}\right\| /\|\tilde{\mathbf{C}}\|$ & $\left\|\tilde{\mathbf{E}}_{G}\right\| /\|\tilde{\mathbf{G}}\|$ & $\left\|\boldsymbol{\epsilon}_{K}\right\| /\left\|\mathbf{A}_{K}\right\|$ & $\left\|\tilde{\mathbf{E}}_{C}\right\| /\|\tilde{\mathbf{C}}\|$ & $\left\|\tilde{\mathbf{E}}_{G}\right\| /\|\tilde{\mathbf{G}}\|$ \\
\hline 1 & 0 & 0 & 0 & 0 & 0 & 0 \\
2 & 0.3319 & 0.0568 & 0.0293 & 0.3164 & 0.0538 & 0.0250 \\
3 & 0.2847 & 0.0591 & 0.0345 & 0.2695 & 0.0580 & 0.0313 \\
4 & 0.3227 & 0.0620 & 0.0396 & 0.3196 & 0.0622 & 0.0376 \\
5 & 0.2995 & 0.0623 & 0.0415 & 0.3129 & 0.0630 & 0.0403 \\
6 & 0.3269 & 0.0626 & 0.0427 & 0.3492 & 0.0635 & 0.0422 \\
7 & 0.3151 & 0.0626 & 0.0432 & 0.3598 & 0.0636 & 0.0430 \\
8 & 0.3404 & 0.0626 & 0.0434 & 0.3951 & 0.0636 & 0.0435 \\
9 & 0.3368 & 0.0626 & 0.0435 & 0.4175 & 0.0636 & 0.0437 \\
10 & 0.3579 & 0.0626 & 0.0436 & 0.4564 & 0.0636 & 0.0438 \\
11 & 0.3602 & 0.0626 & 0.0436 & 0.4892 & 0.0636 & 0.0439 \\
12 & 0.3839 & 0.0626 & 0.0436 & 0.5381 & 0.0636 & 0.0439 \\
13 & 0.3963 & 0.0626 & 0.0437 & 0.5896 & 0.0636 & 0.0439 \\
14 & 0.4340 & 0.0626 & 0.0437 & 0.6691 & 0.0636 & 0.0439 \\
15 & 0.4718 & 0.0626 & 0.0437 & 0.7757 & 0.0636 & 0.0439 \\
\hline
\end{tabular}

\subsection{Multiconductor Transmission Line}

In order to emphasize the general applicability of the illustrated results, this section provides a more complex and realistic application example, pertaining to the multiconductor stripline interconnect whose cross-section is inspired from [15] and depicted in Fig. 6. It consists of eight copper conductors carrying electrical signals and embedded into a dielectric material. Two metal plates at the top and bottom provide the reference for the voltages and the return path for the currents.

The propagation of the voltages and currents along this structure is described in the frequency domain by the following system of LDAEs [21]

$$
\frac{\mathrm{d}}{\mathrm{d} z}\left(\begin{array}{l}
\mathbf{V}(z, f) \\
\mathbf{I}(z, f)
\end{array}\right)+\left(\begin{array}{cc}
\mathbf{0} & \mathbf{R}+j 2 \pi f \mathbf{L} \\
\mathbf{G}+j 2 \pi f \mathbf{C} & \mathbf{0}
\end{array}\right)\left(\begin{array}{l}
\mathbf{V}(z, f) \\
\mathbf{I}(z, f)
\end{array}\right)=\left(\begin{array}{l}
\mathbf{0} \\
\mathbf{0}
\end{array}\right)
$$




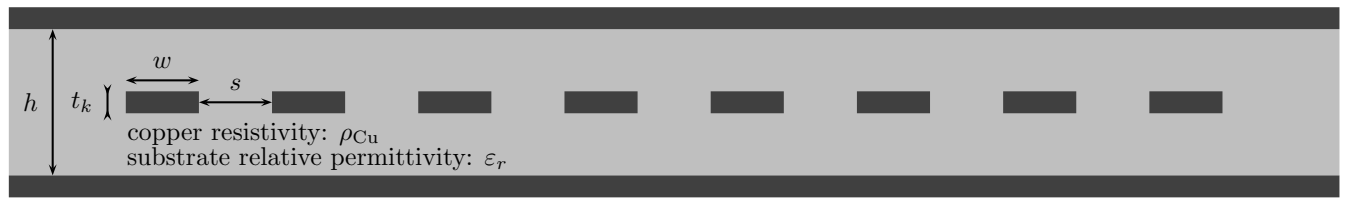

Fig. 6 Cross-section of the eight-conductor stripline interconnect. The deterministic values of the geometrical and material parameters are [15]: $w=0.127 \mathrm{~mm}, s=0.127 \mathrm{~mm}, h=0.508 \mathrm{~mm}, t_{k}=15.24 \mu \mathrm{m}, \rho_{\mathrm{Cu}}=17.241 \mathrm{n} \Omega \cdot \mathrm{m}, \varepsilon_{r}=4$.

where $z \in[0, \ell]$ describes the position along the line length $\ell$ and $f$ is the frequency. Furthermore, $\mathbf{V}(z, f)=$ $\left[V_{1}(z, f), \ldots, V_{8}(z, f)\right]^{t}$ and $\mathbf{I}(z, f)=\left[I_{1}(z, f), \ldots, I_{8}(z, f)\right]^{t}$ are the unknown vectors collecting the voltages and currents along the eight conductors, respectively, whilst $\mathbf{R}, \mathbf{L}, \mathbf{G}, \mathbf{C}$ are matrices describing the electromagnetic behavior and they are computed by means of numerical field simulations. The dielectric medium is assumed to be lossless, hence $\mathbf{G}=\mathbf{0}$ in the following simulations.

Assuming that each conductor is terminated by a resistor $R_{S}$ at $z=0$ and by a capacitor $C_{L}$ at $z=\ell$, the transmission line equation (30) is subject to the boundary conditions

$$
\mathbf{V}(z, f)=\mathbf{V}_{S}-\left.R_{S} \cdot \mathbf{I}(z, f)\right|_{z=0} \quad \mathbf{V}(z, f)=\mathbf{V}_{L}+\left.\frac{1}{j 2 \pi f C_{L}} \mathbf{I}(z, f)\right|_{z=\ell}
$$

where $\mathbf{V}_{S}$ and $\mathbf{V}_{L}$ are the vectors collecting the independent voltage stimuli applied at the two terminations of each conductor.

The following two different random scenarios are considered:

1. The resistivity of the copper conductors and the relative permittivity of the dielectric substrate have uniform variability, and are thus expressed in terms of the uniform random parameter $\xi$ as follows:

$$
\rho_{\mathrm{Cu}}(\xi)=\bar{\rho}\left(1+\Delta_{\rho} \xi\right) \quad, \quad \varepsilon_{r}(\xi)=\bar{\varepsilon}_{r}\left(1+\Delta_{\varepsilon} \xi\right)
$$

with $\bar{\rho}=17.241 \mathrm{n} \Omega \cdot \mathrm{m}, \Delta_{\rho}=90 \%, \bar{\varepsilon}_{r}=4, \Delta_{\varepsilon}=30 \%$. This variation does not affect matrix $\mathbf{L}$ in (30), which is therefore represented by a zero-order PCE $(K=0)$. Furthermore, matrices $\mathbf{R}$ and $\mathbf{C}$ are proportional to $\rho_{\mathrm{Cu}}(\xi)$ and $\varepsilon_{r}(\xi)$, respectively, thus they are represented exactly by first-order PCEs $(K=1)$. Hence, for this scenario the SGM and the PSCM are expected to yield exactly the same PCEs of the line voltages and currents, as a result of Theorem 4.

2. The width of the conductors has a uniform variability, depending on $\xi$ as follows:

$$
w(\xi)=\bar{w}\left(1+\Delta_{w} \xi\right)
$$

with $\bar{w}=0.127 \mathrm{~mm}$ and $\Delta_{w}=90 \%$. However, a variation on $w$ has a nonlinear effect on each of the three matrices $\mathbf{R}, \mathbf{L}$ and $\mathbf{C}$, thus in general the SGM and the PSCM will provide different results for $P>1$.

To confirm the above considerations, a second-order PCE of the line voltages and currents $(P=2)$ is computed with both the SGM and the PSCM. The SGM is implemented as described in [6], i.e., the SGM problem is solved by means of a SPICE-type circuit simulator. In the second scenario, the PCE of the matrices $\mathbf{R}, \mathbf{L}$ and $\mathbf{C}$ is obtained by calculating the integrals in (5) by means of a second-order Gauss quadrature. The PSCM is implemented instead via (14), by sampling the circuit response at the quadrature nodes with the same software.

Figure 7 shows the frequency behavior of the PCE coefficients of the voltages at $z=\ell=10 \mathrm{~cm}$ on the first and second conductor (top panels and bottom panels, respectively), produced by the excitation of the first conductor by a voltage source with an amplitude of $1 \mathrm{~V}$ placed at $z=0$. The left and right panels refer to scenario 1 ) and 2), respectively. It can be once again appreciated that the result of the SGM (solid lines) coincide with the result of the PSCM (markers) only when the dependence of the system matrices on the random parameters is linear, i.e., for the first scenario.

\section{Conclusions}

This paper discusses the relationship between the SGM and the PSCM for LDAEs. It is shown that, under specific yet common assumptions, the PSCM corresponds to an approximate factorization of the SGM. The error introduced by this approximation is investigated and assessed. Specifically, the SGM and the PSCM rigorously coincide only for first-order variations of the system matrices. The approximation error turns out in fact to be zero in that case. The achieved results generalize recent literature on the theory and application of these two methods by extending the discussion to higher-order variations and arbitrary orthogonal polynomial bases. Our findings are illustrated through the simulation of a linear circuit with stochastic elements, and further validated by simulating a stochastic multiconductor transmission line with a SPICE-type circuit simulator. 

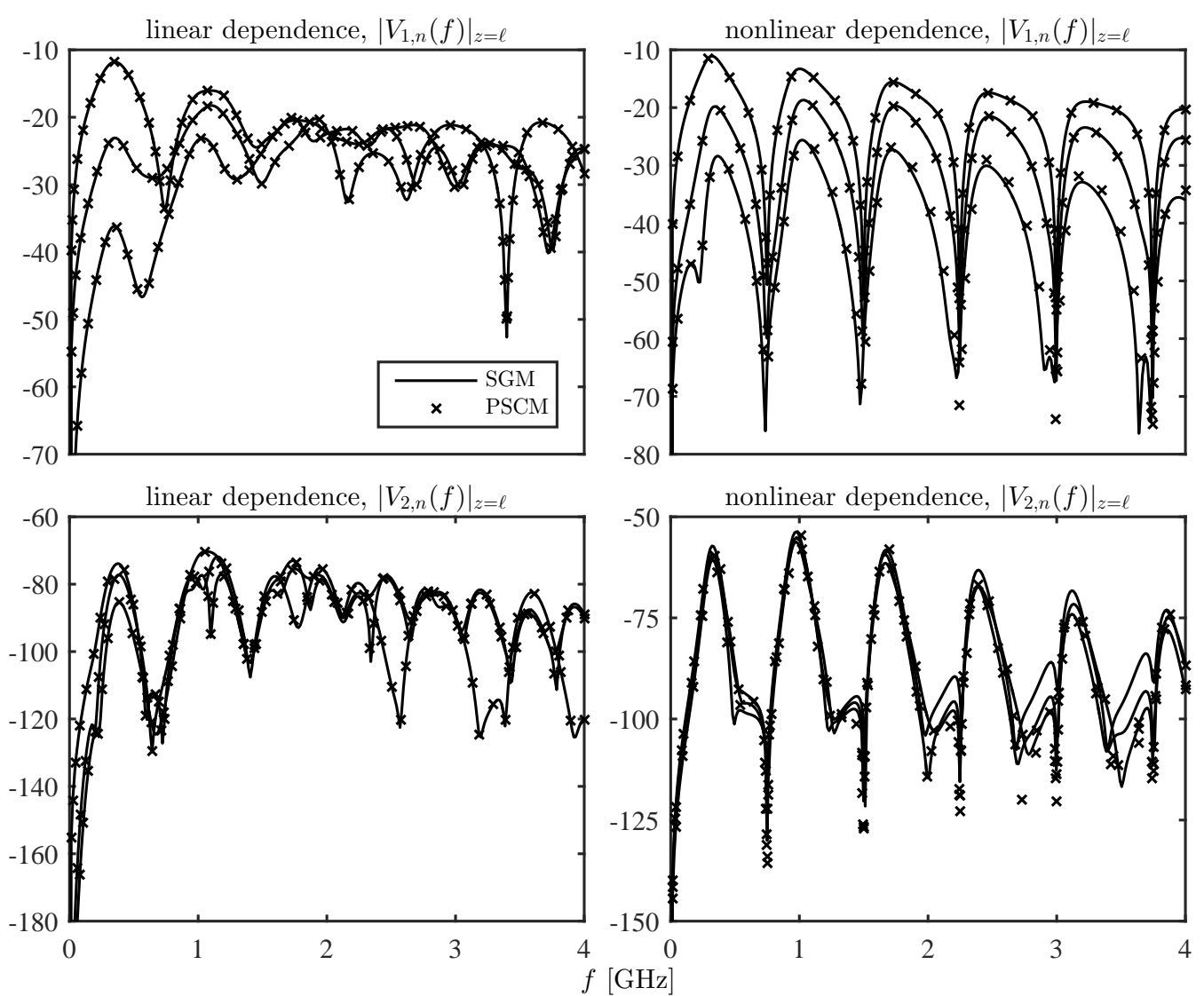

Fig. 7 Frequency behavior at $z=\ell$ of the PCE coefficients of the voltage on the first (top) and second (bottom) conductor for scenario 1) (left) and scenario 2) (right), computed with the SGM (solid lines) and the PSCM (markers).

\section{A Additional proof}

Lemma 2 It holds that $\mathbf{M}=\mathbf{D T D}^{-1}=\mathbf{T}^{t}$.

Proof For the lemma to be true, it must hold that

$$
M_{i j}=D_{i i} T_{i j} \frac{1}{D_{j j}}=T_{j i} \quad i, j=0,1,2 \ldots
$$

Given the form (8) of the tridiagonal matrix $\mathbf{T}$ and the definition of $\mathbf{D}$, the above condition is equivalent to

$$
\frac{1}{\left\|p_{n}\right\|^{2}} \frac{1}{a_{n+1}}\left\|p_{n+1}\right\|^{2}=\frac{c_{n+2}}{a_{n+2}} \Longrightarrow\left\|p_{n+1}\right\|^{2}=c_{n+2} \frac{a_{n+1}}{a_{n+2}}\left\|p_{n}\right\|^{2}, \quad n \geq 0
$$

We now show this is always the case. By using the recurrence relationship (6) and the orthogonality of the polynomials, the squared norm is given by

$$
\begin{aligned}
& \left\|p_{n+1}\right\|^{2}=\left\langle p_{n+1}(\xi), p_{n+1}(\xi)\right\rangle \\
& \quad=a_{n+1}\left\langle\xi p_{n}(\xi), p_{n+1}(\xi)\right\rangle+b_{n+1} \underbrace{\left\langle p_{n}(\xi), p_{n+1}(\xi)\right\rangle}_{=0}-c_{n+1} \underbrace{\left\langle p_{n-1}(\xi), p_{n+1}(\xi)\right\rangle}_{=0}=a_{n+1}\left\langle\xi p_{n}(\xi), p_{n+1}(\xi)\right\rangle .
\end{aligned}
$$

From the recurrence relationship for $p_{n+2}$, it is obtained

$$
\xi p_{n+1}(\xi)=\frac{1}{a_{n+2}} p_{n+2}(\xi)-\frac{b_{n+2}}{a_{n+2}} p_{n+1}(\xi)+\frac{c_{n+2}}{a_{n+2}} p_{n}(\xi) .
$$

Replacing (32) into (31) yields

$$
\left\|p_{n+1}\right\|^{2}=\frac{a_{n+1}}{a_{n+2}} \underbrace{\left\langle p_{n}(\xi), p_{n+2}(\xi)\right\rangle}_{=0}-b_{n+2} \frac{a_{n+1}}{a_{n+2}} \underbrace{\left\langle p_{n}(\xi), p_{n+1}(\xi)\right\rangle}_{=0}+c_{n+2} \frac{a_{n+1}}{a_{n+2}}\left\langle p_{n}(\xi), p_{n}(\xi)\right\rangle=c_{n+2} \frac{a_{n+1}}{a_{n+2}}\left\|p_{n}\right\|^{2}
$$

where the orthogonality of polynomials has again been used.

\section{References}

1. Spence R, Soin RS (1997) Tolerance Design of Electronic Circuits. Imperial College Press, London 
2. Xiu D, Karniadakis GE (2002) The Wiener-Askey polynomial chaos for stochastic differential equations. SIAM J Sci Comput 24(2):619-644

3. Xiu D (2009) Fast numerical methods for stochastic computations: a review. Commun Comput Phys 5(2-4):242-272

4. Ghanem RG, Spanos PD (1991) Stochastic Finite Elements. A Spectral Approach. Springer, New York

5. Strunz K, Su Q (2008) Stochastic formulation of SPICE-type electronic circuit simulation using polynomial chaos. ACM Trans Model Comput Simul 18(4):15:1-15:23

6. Manfredi P, Vande Ginste D, De Zutter D, Canavero FG (2013) Uncertainty assessment of lossy and dispersive lines in SPICEtype environments. IEEE Trans Compon Packag Manuf Techol 3(7):1252-1258.

7. Rufuie MR, Gad E, Nakhla M, Achar R (2014) Generalized Hermite polynomial chaos for variability analysis of macromodels embedded in nonlinear circuits. IEEE Trans Compon Packag Manuf Techol 4(4):673-684

8. Manfredi P, Vande Ginste D, De Zutter D, Canavero FG (2014) Stochastic modeling of nonlinear circuits via SPICE-compatible spectral equivalents. IEEE Trans Circuits Syst I, Reg Papers 61(7):2057-2065

9. Sudret B, Der Kiureghian A (2002) Comparison of finite element reliability methods. Probabilistic Eng Mech 17:337-348

10. Xiu D, Karniadakis GE (2003) Modeling uncertainty in flow simulations via generalized polynomial chaos. J Comput Physics 187:137-167

11. Golub GH, Welsch JH (1969) Calculation of Gauss quadrature rules. Math Comput 221-230

12. Bigoni D, Engsig-Karup AP, Eskilsson C (2016) Efficient uncertainty quantification of a fully nonlinear and dispersive water wave model with random inputs. J Eng Math. doi: 10.1007/s10665-016-9848-8

13. Bäck J, Nobile F, Tamellini L, Tempone R (2011) Stochastic spectral Galerkin and collocation methods for PDEs with random coefficients: a numerical comparison, Spectral and High Order Methods for Partial Differential Equations. Springer, Berlin

14. Pulch R (2014) Stochastic collocation and stochastic Galerkin methods for linear differential algebraic equations. J Comput Appl Math 262:281-291

15. Pham TA, Gad E, Nakhla MS, Achar R (2014) Decoupled polynomial chaos and its applications to statistical analysis of high-speed interconnects. IEEE Trans Compon Packag Manuf Techol 4(10):1634-1647

16. Zhang Z, El-Moselhy TA, Elfadel IM, Daniel L (2013) Calculation of generalized polynomial-chaos basis functions and Gauss quadrature rules in hierarchical uncertainty quantification. IEEE Trans Comput-Aided Des Integr Circuits Syst 32(10):15331545

17. Gradshteyn IS, Ryzhik IM (2007) Table of Integrals, Series, and Products (seventh ed.) Academic Press, San Diego

18. Gaunt JA (1929) The triplets of Helium. Philosoph Trans Roy Soc London 228:151-196

19. Stoer J, Bulirsch R (1993) Introduction to Numerical Analysis (second ed.) Springer, New York.

20. Ho CW, Ruehli A, Brennan P (1975) The modified nodal approach to network analysis. IEEE Trans Circuits Syst 22(6):504-509

21. Paul, CR (1994) Analysis of Multiconductor Transmission Lines. Wiley, New York. 